\title{
Potential inheritance patterns of a prothrombin gene mutation in a 23-year-old female and ethical considerations of a positive diagnosis: a case report
}

Hannah Brand ${ }^{1}$

${ }^{1}$ University of Pittsburgh, School of Dental Medicine

Abstract

Background: Prothrombin, also called Factor II, is a blood clotting protein found in all individuals that is necessary to form blood clots. In most individuals, a balance between bleeding and blood clot formation occurs. However, in individuals with a mutation in the prothrombin gene, the balance is disrupted due to excess production of prothrombin which leads to an increase in blood clot formation (1). Inherited predispositions to blood clot formation are termed hereditary thrombophilia. Prothrombin G20210A mutations are one of the most common hereditary gene associations. Case Description: This report examines the case of a 23-year-old female who has tested positive for the prothrombin gene mutation. The individual has an extensive history of blood clots including 8 deep vein thromboses (DVTs), 4 pulmonary embolisms with one complete infarction, 4 superficial clots, and a miscarried pregnancy attributed to her thrombophilia. The individual has a significant family history of the mutation and takes Coumadin daily for prevention of further clots. Practical and Ethical Implications: Despite a strong familial history of blood clots and related hospitalizations, the parents of the case individual do not want to get their other children tested for the mutation for fear that the children will be denied insurance coverage in their futures. The case individual will likely continue to be on blood thinners indefinitely. This will affect many aspects of her life, including dental treatment, as she will be at an increased risk for bleeding.

\section{Introduction}

Having a mutation in a blood clotting gene such as Prothrombin G20210A, means that an individual has a higher tendency to form blood clots. The prothrombin mutation is found in approximately $2 \%$ of Caucasians in the United States and is the second most common inherited form of thrombophilia (2-4). Given the rarity of the disease, most cases are inherited as heterozygous prothrombin mutations as it is not likely that both parents would possess the mutation, and in heterozygous cases, only one copy of the muta- tion is needed. Homozygous mutations are thought to be more severe than heterozygous. Being heterozygous for the mutation increases the risk of a blood clot by 2-3 times, versus homozygous where the risk is increased by as much as 50 times (3).

The most common complication of having a prothrombin gene mutation is the increased risk for formation of a deep venous thrombosis, or DVT. Typically, these clots form in the leg, but have the potential of traveling through the bloodstream to reach the lungs, known as a pulmonary
Citation: Brand, H. (2017) Potential inheritance patterns of a prothrombin gene mutation in a 23-year-old female and ethical considerations of a positive diagnosis: a case report. Dentistry 3000. 1:a001 doi:10.5195/d3000.2017.69 Received: May 4, 2017

Accepted: May 9, 2017

Published: June 9, 2017

Copyright: (C2017 Brand, H. This is an open access article licensed under a Creative Commons Attribution Work 4.0 United States License. Email: hnb16@pitt.edu (c) BY

ULIS D-Serle?
New articles in this journal are licensed under a Creative Commons Attribution 4.0 United States License.

This journal is published by the University Library System, University of Pittsburgh as part of its D-Scribe Digital Publishing Program and is cosponored by the University of Pittsburgh Press. embolism, or to the brain, a cerebral embolism (5). Women with clotting disorders should be aware that oral contraceptives, hormone therapy, and pregnancy all increase the risk of blood clot formation (1). The prothrombin mutation has been suggested to lead to increased pregnancy complications as well, including preeclampsia, placental abruption, pregnancy loss in all trimesters, and stillbirth (1). Other factors that increase the risk of clot formation are smoking, being overweight or obese, being immobile for long 


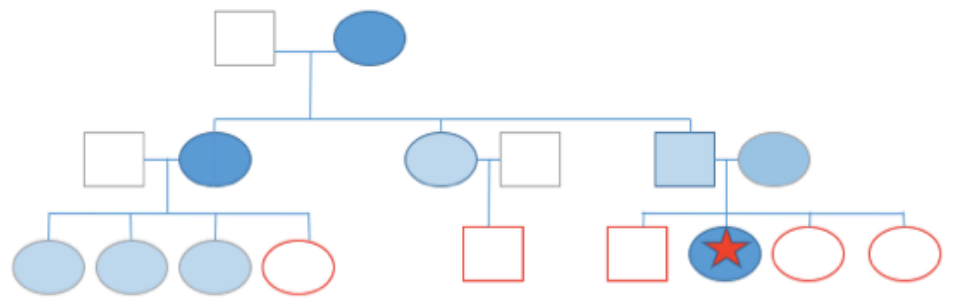

Key: dark blue $=$ homozygous, light blue $=$ heterozygous, white $=$ not tested, star $=$ "Anne"

Figure 1. Hypothetical inheritance patterns of the prothrombin gene mutation in Anne's family. Anne's mothers side of the family has not been tested for the mutation, but because Anne is homozygous recessive, it is likely her mother was a carrier. Given that her paternal grandmother had a history of clots but her father did not, it is likely that her father is a carrier of the trait. Anne and her Aunt have been confirmed as homozygous recessive. Anne's siblings have not been tested for the mutation.

periods of time, and infrequent exercise habits (3).

While refraining from the aforementioned risk factors can sometimes be enough to prevent the formation of a clot, some individuals with the prothrombin mutation still develop clots. Warfarin, an antiplatelet agent, is commonly used to treat these patients. Warfarin works by preventing the proper functioning of Vitamin $\mathrm{K}$ during the formation of clots (2, $6)$. It is important for health care providers, including dentists, to take medications such as Warfarin into consideration when treating patients as they may be at an increased risk for bleeding (7).

The goal of this paper is to report the case of a 23-year-old female with an extensive history of clotting issues stemming from a prothrombin gene mutation. This paper will cover the biological and ethical concerns related to a positive diagnosis of a clotting disorder.

\section{Case Presentation:}

This report examines the case of a 23-year-old female, "Anne" (the individual's name has been changed to protect the individual's identity). Anne has a personal and familial history of blood clots. Anne has had 8 DVTs, 4 pulmonary embolisms with one complete infarction, 4 superficial clots, and delivered a stillborn baby at 5 months. Doctors attributed the miscarriage to her thrombophilia.

Anne's father is Caucasian and her mother is half Japanese and half Caucasian. Her paternal grandmother and grandmother's two sisters all had a history of hospitalizations due to blood clots. Her father's sister tested positive as having the mutation and has also been hospitalized for DVTs and pulmonary embolisms. Anne reported that her father tested negative for the mutation and that her father was not a carrier, but it is likely that he was a heterozygous carrier for the trait. Anne's aunt with a history of blood clots and Anne herself tested positive as homozygous recessive for the trait. Given that many individuals who are heterozygous for the trait never form blood clots and that heterozygous individuals are only at 2-3 times higher risk, it is plausible to think that Anne's parents are carriers of the trait. The extensive history of blood clots and embolisms in Anne's family suggest that Anne is homozygous for the trait, as increased likelihood of developing clots, up to 50 times, and increased severity are reported with homozygous cases (3). A hypothetical pedigree has been proposed (see Figure 1).

\section{Discussion:}

Being diagnosed with a clotting disorder like a prothrombin mutation has many implications that can last a lifetime. Individuals with such mutations are at increased risk for clotting without medication, and are at increased risk for bleeding if on blood thinners such as Warfarin (2). Because factors such as smoking and hormonal birth control significantly increase one's risk for developing a clot, it is advised that individuals with clotting disorders abstain from them.

Anne has 3 siblings from the same parents. It is possible that her siblings may also carry the mutation. Anne has an older 
brother, 26, who is on his own health insurance plan. Her two younger sisters, 16 and 12, are still on their parents' health insurance plan. Anne's dad is unsure whether he wants to have the younger daughters tested for the mutation. While he feels it would be beneficial to know if they have the mutation to better watch for symptoms and avoid risk factors, he fears a positive diagnosis may lead to a lifetime of insurance denial and anxiety related to the condition. The Genetic Information Nondiscrimination Act (GINA) prohibits employers and health care providers from discriminating against individuals because of genetic information (8). It is unknown if the father is unaware of this act, is afraid that this regulation could be overturned in the future, or fears that employers and providers do not always abide by this law.

When seeking medical care and attention, patients with clotting disorders should alert their providers of their condition and of the medications they are taking. Dentists treating patients on Warfarin should be careful to watch for excessive bleeding, though medication adjustments are rarely warranted (2). One could also raise the question of if a patient presents to a dentist with a clotting disorder and a positive history of smoking and/or hormonal birth control, is it the obligation of the dentist to advise the patients of the risks for increased clotting when taking such substances? Based on current literature reporting the significantly heightened risks of clotting with these agents, it is reasonable to think that a dentist should bare this responsibility. The dentist should also counsel the parents on the pros and cons of having the other siblings tested given the potential severity of the disease, while ultimately leaving the decision to the family.

\section{Conclusion}

Anne's medical and familial history of blood clots show the need for awareness of this disorder and the importance of understanding modes of genetic inheritance for determining the risk for other family members. Individuals with clotting disorders should be advised to abstain from factors that increase the risk for clotting, including smoking and hormonal birth control. While it may be considered beneficial to know one's status regarding the mutation to increase awareness and avoid triggering factors, the current state of health care policy in the United States may discourage individuals from getting tested for fear of insurance denial or employment discrimination. It is up to the individual and their family to make these decisions, but regard- less of the decision made, dentists and health care providers have professional and ethical obligations to be aware of and prepared for any potential complications that may arise during treatment.

\section{References}

1. Prothrombin 20210 Mutation (Factor II Mutation); Varga EA, Moll S; Circulation; 2004, July;110(3):e15-8. PMID: 15262854.

2. Yagiela, Dowd, Johnson, et al (2011) Pharmacology and Therapeutics for Dentistry. St. Louis: Mosby Elsevier. 504 p.

3. Lentz, SR; University of Iowa Hospitals and Clinics; 2005. [cited 2017 Mar 15]. Available from https://www.healthcare.uio wa.edu/labs/lentz/Informati on_For_Patients/PDF/Prothr om-

bin\%20Gene\%20Mutation\% 20Brochure.pdf

4. Homozygotes for prothrombin gene 20210 A allele in a thrombophilic family without clinical manifestations of venous thromboembolism; Souto JC, Mateo J, Soria JM, et al.; Haematologica; 1999; 84(7):627-32. PMID: 10406905.

5. Genetics Home Reference; National Institute of Health; U.S. National Library of Medicine; 2008. [cited 2017 Mar 26]. Available from https://ghr.nlm.nih.gov/cond ition/prothrombinthrombophilia\#inheritance 
6. Anticoagulants; The Johns Hopkins Lupus Center; 2017. [cited 2017 Mar 26]. Available from https://www.hopkinslupus.o rg/lupustreatment/commonmedicationsconditions/anticoagulants/

7. Anticoagulant and Antiplatelet Medications and Dental Procedures; The American Dental Association; 2015. [cited 2017 Mar 28] Available from

http://www.ada.org/en/me mber-center/oral-healthtopics/anticoagulantantiplatelet-medicationsand-dental-

8. Genetic Information Nondiscrimination Act; U.S. Department of Health and Human Services; 2017. [cited 2017 Mar 28]. Available from https://www.hhs.gov/hipaa/ for-professionals/specialtopics/geneticinformation/index.html 